\section{Avaliação da coordenação do cuidado na atenção primária à saúde: comparando o PMAQ-AB (Brasil) e referências internacionais}

\author{
Assessment of the coordination of primary \\ healthcare: comparison of PMAQ-AB (Brazil) and \\ international references
}

Maria Jesus Barreto Cruz ${ }^{1}$

Alaneir de Fátima dos Santos 1

César Macieira 1

Daisy Maria Xavier de Abreu 1

Antônio Thomaz Gonzaga da Matta Machado 1

Eli Iola Gurgel Andrade 1

doi: 10.1590/0102-311X00088121

\section{Resumo}

O objetivo deste estudo foi comparar os resultados obtidos para a coordenação do cuidado a partir do Programa Nacional de Melhoria do Acesso e da Qualidade da Atenção Básica (PMAQ-AB), com os parâmetros adotados pelo Atlas de Medidas de Coordenação do Cuidado e pelo Observatório Europeu de Politicas e Sistemas de Saúde. Foi realizado estudo transversal, com base no banco de dados do 3 o ciclo do PMAQ-AB. Foram criadas três tipologias de coordenação do cuidado: PMAQ-AB, Atlas e Observatório. O teste qui-quadrado foi aplicado para comparar as proporções; os testes de Kruskal-Wallis $e$ de Nemenyi para verificar e identificar eventuais diferenças entre as tipologias. O nível de significância foi de 5\%. Foram avaliadas 35.350 equipes que realizaram alguma atividade de coordenação do cuidado. Observou-se diferença significativa $(p<0,001)$, entre os níveis de coordenação, com maior percentual entre o nível alto e médio nos três instrumentos, PMAQ-AB (56,07\% e 38,35\%), Atlas (52,63\% e 40,66\%) e o Observatório $(44,82 \%$ e 43,98\%). Na comparação dos indicadores, houve diferença significativa $(p<0,001)$ entre as tipologias. Para o Brasil, na tipologia PMAQ-AB, todos os estratos exibiram maior percentual entre o nível alto e médio; no Atlas, o estrato 1 destacouse no nível médio $(43,81 \%)$ e, no Observatório, predominou o nível alto. $\mathrm{Na}$ comparação dos indicadores por estratos, pelo menos um estrato diferiu dos demais $(p<0,001)$. O 6 se distinguiu dos demais $(p<0,001)$, e o 1 diferiu de todos $(p<0,001)$, exceto do $2(p>0,05)$. Os níveis de coordenação do cuidado diferenciaram-se entre os instrumentos utilizados. Altos e médios níveis foram identificados, demonstrando a necessidade de estudos adicionais.

Qualidade, Acesso e Avaliação da Assistência à Saúde; Atenção Primária à Saúde; Estudo Comparativo; Garantia da Qualidade dos Cuidados de Saúde; Pesquisas sobre Serviços de Saúde

\section{Correspondência}

M. J. B. Cruz

Faculdade de Medicina, Universidade Federal de Minas Gerais. Av. Prof. Alfredo Balena 190, Belo Horizonte, MG 30130-100, Brasil.

maria_enfermagem@yahoo.com.br

1 Faculdade de Medicina, Universidade Federal de Minas Gerais, Belo Horizonte, Brasil. 


\section{Introdução}

Estudos envolvendo a coordenação de cuidados em saúde são de grande importância nos sistemas contemporâneos. Contudo esta é uma tarefa desafiadora, em parte pela dificuldade de entendimento do conceito, por ser um produto de várias organizações e pela dificuldade em distingui-la de outros aspectos no processo de cuidados em saúde 1 . Na tentativa de entender a coordenação do cuidado, pesquisadores do Reino Unido, considerando a opinião de 50 especialistas na área, testaram três hipóteses diferentes e verificaram que nenhuma pode ser descartada, sendo elas: problemas com o modelo; problemas de implementação e problemas de avaliação 2 .

$\mathrm{Na}$ literatura, várias são as definições disponíveis para o atributo. Segundo Starfield 3, a coordenação é um estado de estar em harmonia numa ação ou esforço comum, e o fator marcante é a existência e o reconhecimento de informações sobre todo o processo de cuidado. Já ao considerar a multidimensionalidade do atributo, McDonald et al. ${ }^{4}$ afirmam que a coordenação do cuidado é a organização deliberada de atividades, desenvolvidas por meio do estabelecimento de vínculo entre os diversos participantes do processo de cuidado, o que inclui o paciente e visa atender a necessidades específicas, utilizando trocas qualificadas de informação.

Uma característica marcante é que a coordenação do cuidado pode ser reconhecida nos mais diversos cenários da atenção à saúde. Baseado no dinamismo do envolvimento entre os atores, na influência do ambiente no desenvolvimento das ações e na intensa variedade de serviços que atuam em uma interação contínua, o termo "ecossistemas de cuidado" vem se tornando comum, podendo ser importante para compreender a coordenação do cuidado 5.

Diversas pesquisas comprovam que serviços de saúde bem coordenados garantem mais segurança ao usuário, fortalece vínculos e reduz gastos ao sistema 6,7,8. Além disso, falhas ainda acontecem com frequência, sobretudo na transição de informações entre os serviços, causando, entre outros problemas, erros de medicação, duplicação de exames, readmissões hospitalares e lacunas na oferta de cuidados preventivos $6,9,10$.

A dificuldade em medir essa complexa construção tem limitado o entendimento da influência da coordenação nos resultados de avaliações de políticas e ações de saúde e também impedido avanços diretos no cuidado 2,11. Trabalhos vêm sendo desenvolvidos com o objetivo de construir instrumentos que permitam a avaliação desse atributo nas realidades assistenciais. Destaca-se, entre eles, o Atlas de Medidas de Coordenação do Cuidado, criado pela Agência de Pesquisa e Qualidade em Saúde dos Estados Unidos (Agency for Healthcare Research and Quality - AHRQ), em 2010. O documento fornece um compêndio de avaliações de coordenação do cuidado nos mais diferenciados tipos de serviço e países, considerando a perspectiva de pacientes, cuidadores, profissionais e sistemas de saúde 4 . Sua formulação absorve as experiências advindas dos Estados Unidos com sistema de saúde de caráter liberal, mas, por constituir-se a partir do grupo estruturado inicialmente por Barbara Starfield, contribui com reflexões importantes sobre a coordenação de cuidado na atenção primária à saúde (APS). É considerado um avanço por incorporar concepções multidimensionais 11,12.

A versão inicial do Atlas era centrada em medidas de coordenação do cuidado de modo geral e apresentava poucos instrumentos destinados a APS. Atualizado em 2014, em um cenário de crescente interesse pela coordenação do cuidado e o reconhecimento de que a APS desempenha um papel central no desenvolvimento do atributo em todos os ambientes, o Atlas passou a oferecer maior diversidade de medidas voltadas a esse nível de atenção 4. Diante da relevância do tema, a AHQR vem dando continuidade ao trabalho do Atlas e desenvolveu, em 2016, a pesquisa Medida de Qualidade da Coordenação de Cuidados para a Atenção Básica 13.

A pesquisa realizada pelo Observatório Europeu de Políticas e Sistemas de Saúde, que resultou na publicação do documento Construindo Cuidados Primários em uma Europa em Mudança 8, em 2015, também é identificada como importante referência na avaliação da coordenação do cuidado. $O$ trabalho visava entender como a APS é organizada e ofertada em 31 países europeus que relataram possuir este modelo de atenção ${ }^{8}$. Foram utilizados 115 indicadores relacionados à estrutura (governança, condições econômicas e desenvolvimento de recursos humanos), ao processo (acessibilidade, continuidade, coordenação e resolução) e aos resultados (qualidade, eficiência e equidade) 14.

Dentre os resultados obtidos, o estudo apresenta uma análise sobre a importância de uma APS forte para o desempenho do sistema de saúde, o mapeamento e a comparação nos países pesquisados 8 . 
Demonstrou a complexidade e a variabilidade da APS na Europa, conformando dois grupos de países, um com sistema fortemente ancorado na APS e outro com sistema fraco e desequilibrado 14. Possibilitou entender a potencialidade da APS em coordenar o uso de serviços de saúde, evidenciando fragilidades e as capacidades de cada país, fornecendo elementos úteis para a avaliação da coordenação do cuidado neste nível assistencial 8,15.

O Instrumento de Avaliação da Atenção Primária (PCATool - Primary Care Assessment Tool), criado no Centro de Políticas de Atenção Primária Johns Hopkins (Johns Hopkins Primary Care Policy Center - Estado Unidos), é largamente utilizado para avaliar os atributos da APS, tendo como objetivo medir a presença e a extensão dos atributos essenciais e derivados. No Brasil, este é o instrumento mais utilizado para a avaliação da coordenação do cuidado. Importante destacar que estudos utilizando PCATool, quanto à coordenação do cuidado, avaliam como atributo ainda em processo de construção no país $16,17,18,19$.

No Brasil, o Programa Nacional de Melhoria do Acesso e da Qualidade da Atenção Básica (PMAQ-AB), criado pelo Ministério da Saúde em 2011, é uma ferramenta que permite a avaliação da coordenação do cuidado, prevendo a participação de gestores, profissionais e usuários 20,21. Propicia informações que possibilitam um panorama da real situação do atributo no país, considerando regiões, estados e estratos 22. Com base em dados do PMAQ-AB, Fausto et al. 23, ao avaliarem a coordenação do cuidado, identificaram a presença de mecanismos como continuidade informacional, ordenamento de fluxos assistenciais e coordenação do cuidado clínico que são considerados fundamentais para a integração da rede assistencial. Contudo, consideram que ainda são incipientes ações nesta direção.

Em pesquisa conduzida por Souza et al. 22, foram identificados elementos relacionados à coordenação do cuidado, como também os que demandavam maior nível de coordenação pelas equipes. Em outro trabalho, as barreiras ao compartilhamento de registros afetaram a coordenação do atendimento para muitos dos pacientes entrevistados, destacando a necessidade crítica de melhorar o fluxo de informações em saúde e a interoperabilidade 24 .

Estudo considerando uma avaliação entre o I e o II ciclo do PMAQ-AB em relação aos atributos da APS, envolvendo dados das equipes de saúde da família (EqSF), unidades e usuários, identificou que os atributos com pior avaliação foram longitudinalidade e coordenação, por dificuldades de acesso às consultas especializadas e registros insuficientes 25 . Em um ensaio teórico sobre coordenação do cuidado no Brasil, foram identificados alguns aspectos que são importantes para a estruturação da coordenação pela APS - posição da EqSF na rede assistencial; integração entre níveis assistenciais e interfaces com a regulação assistencial 18.

Diante do exposto, o objetivo deste artigo é comparar os resultados brasileiros obtidos a partir do PMAQ-AB para o atributo coordenação do cuidado, com os parâmetros adotados pelo Atlas de Medidas de Coordenação do Cuidado e pelo Observatório Europeu de Políticas e Sistemas de Saúde.

\section{Métodos}

Estudo transversal, com abordagem quantitativa, tendo como fonte o banco de dados do 3 o ciclo do PMAQ-AB, realizado entre os anos de 2018 e 2019, contemplando 38.865 equipes de atenção básica distribuídas em todo o território nacional.

Inicialmente, as questões relativas à coordenação do cuidado foram identificadas no instrumento de avaliação externa do Módulo II, que contém a entrevista do profissional de saúde sobre o processo de trabalho da equipe e a organização do cuidado, além da verificação de documentos relacionados à unidade de saúde. Para a seleção das questões, foi considerado o conceito amplo de coordenação do cuidado, referenciado em estudos nacionais e internacionais, abrangendo aspectos gerenciais à oferta de cuidados em todas as áreas contempladas pelo PMAQ-AB: saúde mental, tuberculose, hipertensão, diabetes, obesidade, hanseníase, saúde da mulher, saúde da criança e saúde na escola.

A identificação das questões foi norteada pelas seguintes atividades de coordenação do cuidado: ações e mecanismos de comunicação; apoio comunitário; apoio para tomada de decisão; compartilhamento de informações entre os envolvidos no processo de cuidado; estabelecimento e/ou compartilhamento de responsabilidade; estratificação de risco do território e das condições de atenção; implantação e utilização do e-SUS e prontuário eletrônico; mecanismos de referência e contrarreferência; 
organização da agenda; planejamento de ações; processo de trabalho entre as equipes de APS e as equipes de apoio matricial e institucional; registro de informações; reuniões; utilização de protocolos clínicos e uso de sistemas de informação. Foram identificadas as equipes que relataram realizar algum tipo de atividade de coordenação, totalizando 35.350 equipes.

A partir das potencialidades do PMAQ-AB, foram elaboradas três tipologias de coordenação do cuidado na APS no Brasil: coordenação do cuidado segundo o PMAQ-AB; coordenação do cuidado segundo o Atlas de Medidas de Coordenação do Cuidado (Atlas) e segundo o Observatório Europeu de Políticas e Sistemas de Saúde (Observatório). Tais instrumentos foram utilizados por se constituírem em referências centrais para a avaliação do atributo coordenação, em sistemas de APS no mundo. Para a construção da tipologia, foram utilizados os aspectos teóricos e práticos da coordenação do cuidado. O Quadro 1 descreve o percurso metodológico para a seleção das questões.

Dentre os domínios/atividades de coordenação do cuidado propostos por cada instrumento, foram identificados os que teriam similaridade com o PMAQ-AB, a saber:

(1) Atlas: estabelecer ou negociar responsabilidade; comunicar (comunicação interpessoal e transferência de informação); facilitar as transições; avaliar as necessidades e os objetivos; criar um plano proativo de cuidados; monitorar, acompanhar e responder às mudanças; apoiar os objetivos de autogestão; link para recursos da comunidade e alinhar recursos com necessidades de pacientes e população 4 .

(2) Observatório: manutenção de registros médicos; sistemas eletrônicos de suporte clínico; sistema de referência; procedimentos de informação clínica de contrarreferência; comunicação entre especialista/clínico geral/médico de família/ginecologista/obstetra; cooperação dentro da APS; colaboração dentre APS e atenção secundária; pesquisas em saúde comunitária 8 .

Do total de 117 questões selecionadas no PMAQ-AB, 90 apresentaram correlação com os domínios/atividades de coordenação propostos pelo Atlas, 58 apresentaram correlação com os domínios/ atividades de coordenação propostos pelo Observatório e 19 questões não apresentaram correlação com nenhum dos dois instrumentos.

A seguir, cada questão contida nos blocos foi categorizada do seguinte modo: respostas negativas receberam pontuação 0 (zero) e, caso positivas, 1 (um); vale ressaltar que respostas do tipo "não se aplica", "não sabe/não respondeu" não tiveram valores atribuídos. Em continuidade, foi criado um nível de coordenação, estimado pela razão entre o somatório das atividades de coordenação do cuidado (desenvolvidas pelas equipes) e o total de questões referentes à coordenação, sem ponderação de pesos entre as variáveis, sendo: coordenação baixa (0 a 49,9\%), média (50 a 75\%) e alta (maior que 75\%). Para atender aos objetivos do estudo, foram criados, separadamente, níveis de coordenação do cuidado para as três tipologias de avaliação.

A descrição das tipologias foi feita por intermédio de frequências absolutas e relativas, considerando o país e os estratos.

Os estratos foram criados pelo Ministério da Saúde, com a intenção de garantir maior equidade na comparação entre as equipes no processo de certificação, considerando aspectos socioeconômicos e demográficos. A definição dos estratos foi feita com base em índice que varia de 0 a 10 e porte populacional. O índice é composto por cinco indicadores: Produto Interno Bruto (PIB) per capita; percentual da população com plano de saúde; percentual da população com Bolsa Família; percentual da população em extrema pobreza e densidade demográfica.

Para verificar se havia diferenças significativas entre as tipologias categóricas construídas e seus resultados, considerando país e estratos, foi aplicado o teste qui-quadrado realizando uma comparação das proporções (homogeneidade) dos indicadores 26. Para apurar a avaliação, foi realizada, a seguir, análise da distribuição dos indicadores via teste de Anderson-Darling 27, verificando a suposição de normalidade. Após constatar não se tratar de distribuição normal ( $<<0,001)$, foi aplicado o teste não paramétrico de Kruskal-Wallis, com o objetivo de verificar existência de diferenças entre os indicadores construídos e estratos e país 28.

As comparações múltiplas foram realizadas mediante teste de Nemenyi, ou seja, dado que houve diferenças significativas entre os estratos e país de modo geral (via teste de Kruskal-Wallis), o teste de Nemenyi identifica onde ocorreram diferenças executando uma comparação dois a dois 28. O nível de significância utilizado nestas análises foi de $5 \%$.

O estudo foi aprovado pelo Comitê de Ética em Pesquisa da Universidade Federal de Minas Gerais (registro 28.804, em 30 de maio de 2012). 


\section{Quadro 1}

Percurso metodológico para a seleção das questões.

\begin{tabular}{|c|c|c|c|}
\hline PASSO 1 & \multicolumn{3}{|l|}{ Identificação do conceito de coordenação do cuidado } \\
\hline PASSO 2 & \multicolumn{3}{|l|}{ Identificação dos domínios/atividades de coordenação do cuidado no Atlas e no Observatório } \\
\hline PASSO 3 & \multicolumn{3}{|l|}{ Busca no instrumento de avaliação externa pelas questões de coordenação do cuidado } \\
\hline PASSO 4 & \multicolumn{3}{|c|}{$\begin{array}{l}\text { Correlação das questões encontradas com os domínios/atividades de coordenação do cuidado identificadas no Atlas e no } \\
\text { Observatório }\end{array}$} \\
\hline \multicolumn{2}{|r|}{ QUESTÕES IDENTIFICADAS NO INSTRUMENTO DE AVALIAÇÃO EXTERNA - PMAQ-AB } & ATLAS & OBSERVATÓRIO \\
\hline \multicolumn{2}{|c|}{ A equipe recebe apoio de outros profissionais para a resolução de casos complexos? Quais? } & $x$ & $x$ \\
\hline \multicolumn{2}{|c|}{ No último ano, a equipe participou de ações de educação permanente? } & $x$ & \\
\hline \multicolumn{2}{|c|}{ Quais dessas ações a equipe participa ou participou no último ano? } & $x$ & $\mathrm{x}$ \\
\hline \multicolumn{2}{|c|}{ Essas ações de educação permanente contemplam as demandas e necessidades da equipe? } & & $\mathrm{x}$ \\
\hline \multicolumn{2}{|c|}{ Sobre a integração ensino-serviço na saúde, a sua equipe conta com a participação de quais suportes? } & $\mathrm{x}$ & $\mathrm{x}$ \\
\hline \multicolumn{2}{|c|}{ A equipe utiliza o Telessaúde? Utiliza o Telessaúde para:/Utiliza o 0800 do Telessaúde? } & $\mathrm{x}$ & $\mathrm{x}$ \\
\hline \multicolumn{2}{|c|}{ Essas ações contemplam as demandas e necessidades da equipe? } & $\mathrm{x}$ & \\
\hline \multicolumn{2}{|c|}{ Como a equipe avalia o atendimento recebido no $0800 ?$} & $x$ & \\
\hline \multicolumn{2}{|c|}{$\begin{array}{l}\text { A gestão considerou critérios de risco e vulnerabilidade para definir da quantidade de pessoas sob } \\
\text { responsabilidade da equipe? }\end{array}$} & $\mathrm{x}$ & $x$ \\
\hline \multicolumn{2}{|c|}{ Os prontuários estão organizados por núcleos familiares? Existe documento que comprove? } & $\mathrm{x}$ & \\
\hline \multicolumn{2}{|c|}{ Como são registradas as informações de saúde? } & $\mathrm{x}$ & \\
\hline \multicolumn{2}{|c|}{ Qual o tipo de prontuário eletrônico? Existe documento que comprove? } & $x$ & \\
\hline \multicolumn{2}{|c|}{ Sua equipe recebeu qualificação para utilizar a classificação internacional para atenção primária? } & $x$ & \\
\hline \multicolumn{2}{|c|}{ Sua equipe recebeu qualificação ou capacitação para utilização do prontuário eletrônico? } & $\mathrm{x}$ & \\
\hline \multicolumn{2}{|c|}{ Quando a equipe tem dúvidas sobre o prontuário eletrônico, tem um canal para sanar dúvida? } & $x$ & \\
\hline \multicolumn{2}{|c|}{ Quais canais são utilizados? } & $x$ & $x$ \\
\hline \multicolumn{2}{|c|}{ Sobre o registro clínico orientado a problemas no prontuário eletrônico, quais itens sua equipe utiliza? } & $x$ & \\
\hline \multicolumn{2}{|c|}{ A sua equipe realiza reunião de equipe? } & $x$ & $x$ \\
\hline \multicolumn{2}{|c|}{ Qual a periodicidade das reuniões? } & & $x$ \\
\hline \multicolumn{2}{|c|}{ A equipe realiza atividade para o planejamento das ações? Existe documento que comprove? } & $\mathrm{x}$ & $\mathrm{x}$ \\
\hline \multicolumn{4}{|c|}{ Qual a periodicidade? } \\
\hline A equipe $r$ & nitoramento e análise dos indicadores e informações de saúde? & $x$ & $x$ \\
\hline Os resulta & sados no PMAQ-AB foram considerados na organização do processo de trabalho? & & $\mathrm{x}$ \\
\hline No último & alizado algum processo de autoavaliação pela equipe? & & \\
\hline Recebe ap & cional da Secretaria Municipal de Saúde para discutir o processo de trabalho? & $\mathrm{x}$ & $x$ \\
\hline Como a ec & a o trabalho com o Apoiador Institucional? & & \\
\hline A gestão c & a informações para análise de situação de saúde da população? & & $x$ \\
\hline Quando o & recisa agendar uma consulta, qual(is) é(são) a(s) possibilidade(s)? & $x$ & $x$ \\
\hline Em relaçã & da de cuidado continuado, como é realizada a marcação de consulta? & $x$ & \\
\hline O usuário & dade com a consulta marcada, quando não é preciso atender no mesmo dia? & & $x$ \\
\hline A equipe $r$ & tudo da demanda espontânea nos últimos 12 meses? Qual o fluxo de acolhimento? & $x$ & $x$ \\
\hline $\begin{array}{l}\text { A equipe } \\
\text { Existe doc }\end{array}$ & $\begin{array}{l}\text { ocolos/critérios para orientação das condutas dos casos atendidos no acolhimento? } \\
\text { ue comprove? }\end{array}$ & $\mathrm{x}$ & $\mathrm{x}$ \\
\hline Os profiss & fazem o acolhimento foram capacitados para o uso dos protocolos? & $x$ & $x$ \\
\hline A equipe & il acesso ao usuário para? & $x$ & $x$ \\
\hline A equipe $t$ & a serviço para remoção do usuário quando necessário? & & \\
\hline $\begin{array}{l}\text { A equipe } \\
\text { de serviço }\end{array}$ & $\begin{array}{l}\text { umento orientador para ações e os procedimentos ofertados ao usuário (p.ex.: carteira } \\
\text { documento que comprove? }\end{array}$ & $x$ & \\
\hline Existe flux & inicação institucionalizado entre a equipe e a atenção especializada? Qual? & $x$ & $x$ \\
\hline
\end{tabular}

(continua) 
Quadro 1 (continuação)

\begin{tabular}{|c|c|c|}
\hline QUESTÕES IDENTIFICADAS NO INSTRUMENTO DE AVALIAÇÃO EXTERNA - PMAQ-AB & ATLAS & OBSERVATÓRIO \\
\hline $\begin{array}{l}\text { Com que frequência os profissionais de atenção básica e especialistas entram em contato para trocar } \\
\text { informações sobre os pacientes encaminhados? }\end{array}$ & $\mathrm{x}$ & $\mathrm{x}$ \\
\hline Indique para quais das condições abaixo existem referências e fluxos definidos: & $\mathrm{x}$ & $\mathrm{x}$ \\
\hline Existe central de regulação para o encaminhamento dos usuários para os demais pontos de atenção? & & $\mathrm{x}$ \\
\hline Quais centrais de marcação disponíveis? & $\mathrm{x}$ & $\mathrm{x}$ \\
\hline Quando um usuário necessita ser encaminhado para consulta especializada, quais são as formas? & $\mathrm{x}$ & $\mathrm{x}$ \\
\hline A equipe possui registro de mulheres com exame citopatológico atrasado? Existe documento que comprove? & $\mathrm{x}$ & $\mathrm{x}$ \\
\hline $\begin{array}{l}\text { A equipe possui registro de mulheres com exame citopatológico, de mamografia e/ou ultrassom mamário } \\
\text { alterado? Existe documento que comprove? }\end{array}$ & $\mathrm{x}$ & $\mathrm{x}$ \\
\hline A equipe utiliza protocolos para identificação precoce/rastreamento? Quais? & $\mathrm{x}$ & \\
\hline \multicolumn{3}{|l|}{ A equipe realiza busca ativa das seguintes situações: } \\
\hline A equipe realiza seguimento das mulheres após tratamento na atenção especializada? & $\mathrm{x}$ & \\
\hline A equipe utiliza estratégias de sensibilização para realização do exame citopatológico e de mamas? & $\mathrm{x}$ & \\
\hline A equipe utiliza protocolos para estratificação de risco das gestantes? & $\mathrm{x}$ & \\
\hline $\begin{array}{l}\text { A equipe organiza as ofertas de serviço e encaminhamentos (consultas, exames) das gestantes conforme } \\
\text { avaliação e classificação de risco e vulnerabilidade? }\end{array}$ & $\mathrm{x}$ & \\
\hline A equipe possui registro do número de gestantes de alto risco? A equipe utiliza caderneta da gestante? & & $\mathrm{x}$ \\
\hline \multicolumn{3}{|l|}{ Tem cópia das cadernetas das gestantes? No acompanhamento da gestante há registro de: } \\
\hline \multicolumn{3}{|l|}{ Que ações a equipe realiza para garantir a consulta de puerpério até uma semana após o parto? } \\
\hline $\begin{array}{l}\text { A equipe utiliza protocolos voltados para atenção a crianças menores de dois anos? A equipe possui } \\
\text { cadastramento atualizado de crianças até dois anos do território? }\end{array}$ & $\mathrm{x}$ & \\
\hline \multicolumn{3}{|l|}{ A equipe utiliza a caderneta de saúde da criança?/Tem espelho das cadernetas de saúde da criança? } \\
\hline No acompanhamento das crianças do território, há registro sobre: & $\mathrm{x}$ & \\
\hline A equipe acompanha casos de violência familiar junto como profissionais de outro serviço? & $\mathrm{x}$ & \\
\hline \multicolumn{3}{|l|}{ A equipe realiza busca ativa das crianças? } \\
\hline A equipe desenvolve ações de promoção do aleitamento exclusivo para crianças até seis meses? & $\mathrm{x}$ & \\
\hline $\begin{array}{l}\text { A equipe desenvolve ações de estímulo à introdução de alimentos saudáveis e aleitamento materno } \\
\text { continuado a partir dos seis meses da criança? }\end{array}$ & $\mathrm{x}$ & \\
\hline A equipe utiliza protocolos para estratificação de risco dos usuários com HAS? & $\mathrm{x}$ & \\
\hline A equipe avalia a presença de comorbidades e fatores de risco cardiovascular dos usuários com HAS? & $\mathrm{x}$ & $\mathrm{x}$ \\
\hline A equipe utiliza alguma ficha de cadastro ou acompanhamento de pessoas com HAS? & $\mathrm{x}$ & \\
\hline \multicolumn{3}{|l|}{ A equipe realiza acompanhamento de usuários com diagnóstico de doença cardíaca? } \\
\hline A equipe programa consultas e exames de pessoas com HAS em função da estratificação dos casos? & $\mathrm{x}$ & \\
\hline A equipe possui registro dos usuários com HAS com maior risco? Existe documento que comprove? & $\mathrm{x}$ & $\mathrm{x}$ \\
\hline $\begin{array}{l}\text { A equipe coordena a fila de espera e acompanhamento dos usuários com HAS que necessitam de consultas } \\
\text { e exames em outros pontos de atenção? }\end{array}$ & $\mathrm{x}$ & \\
\hline $\begin{array}{l}\text { A equipe possui o registro dos usuários com HAS de maior risco/gravidade encaminhados para outro ponto } \\
\text { de atenção? Existe documento que comprove? }\end{array}$ & $\mathrm{x}$ & $\mathrm{x}$ \\
\hline A equipe utiliza protocolos para estratificação de risco dos usuários com diabetes mellitus? & $\mathrm{x}$ & \\
\hline $\begin{array}{l}\text { A equipe possui registro de usuários com diabetes mellitus com maior risco? Existe documento que } \\
\text { comprove? }\end{array}$ & $\mathrm{x}$ & $\mathrm{x}$ \\
\hline A equipe utiliza alguma ficha de cadastro ou acompanhamento de pessoas com diabetes mellitus? & $\mathrm{x}$ & $\mathrm{x}$ \\
\hline $\begin{array}{l}\text { A equipe coordena a fila de espera e acompanhamento dos usuários com diabetes mellitus que necessitam } \\
\text { de consultas e exames em outros pontos de atenção? }\end{array}$ & $\mathrm{x}$ & $\mathrm{x}$ \\
\hline $\begin{array}{l}\text { A equipe possui registro dos usuários com diabetes mellitus encaminhados? Existe documento que } \\
\text { comprove? }\end{array}$ & $\mathrm{x}$ & $\mathrm{x}$ \\
\hline $\begin{array}{l}\text { A equipe programa as consultas e exames de pessoas com diabetes mellitus em função da estratificação dos } \\
\text { casos? }\end{array}$ & $\mathrm{x}$ & $\mathrm{x}$ \\
\hline
\end{tabular}

(continua) 
Quadro 1 (continuação)

\begin{tabular}{|c|c|c|}
\hline QUESTÕES IDENTIFICADAS NO INSTRUMENTO DE AVALIAÇÃO EXTERNA - PMAQ-AB & ATLAS & OBSERVATÓRIO \\
\hline \multicolumn{3}{|l|}{ Após identificação de usuário com obesidade (IMC $\left.\geq 30 \mathrm{~kg} / \mathrm{m}^{2}\right)$, a equipe realiza alguma ação? Qual? } \\
\hline \multicolumn{3}{|l|}{ Aciona equipe de Apoio Matricial para apoiar o acompanhamento deste usuário na UBS? } \\
\hline \multicolumn{3}{|l|}{ Encaminha para serviço especializado? } \\
\hline A equipe identifica as áreas de maior risco de transmissão vetorial? Existe documento que comprove? & $\mathrm{x}$ & \\
\hline A gestão municipal ofertou para equipe? & $\mathrm{x}$ & \\
\hline $\begin{array}{l}\text { A equipe realiza classificação de risco dos pacientes com suspeita de dengue, Zika vírus, febre Chikungunya e } \\
\text { febre amarela, conforme os fluxogramas? }\end{array}$ & $\mathrm{x}$ & $x$ \\
\hline \multicolumn{3}{|l|}{ A equipe solicita o hemograma com contagem de plaquetas para o paciente com suspeita de dengue? } \\
\hline A equipe possui registro dos usuários do território? & $x$ & $\mathrm{x}$ \\
\hline A equipe possui o registro dos seguintes usuários de maior risco encaminhados: & $\mathrm{x}$ & $\mathrm{x}$ \\
\hline A equipe possui registro do número de pessoas com deficiência? Existe documento que comprove? & $\mathrm{x}$ & $\mathrm{x}$ \\
\hline $\begin{array}{l}\text { A equipe possui o registro dos usuários com deficiência em acompanhamento por outras equipes/serviços? } \\
\text { Existe documento que comprove? }\end{array}$ & $x$ & $\mathrm{x}$ \\
\hline Com quais serviços a equipe conta para o acompanhamento das pessoas com deficiência? & $\mathrm{x}$ & \\
\hline \multicolumn{3}{|l|}{ A Secretaria Municipal de Saúde fornece normas para o desenvolvimento do trabalho dos ACS? } \\
\hline As famílias são visitadas de forma distinta de acordo com avaliações de risco e vulnerabilidade? & $\mathrm{x}$ & \\
\hline A equipe desenvolve ações voltadas à promoção à saúde? Quais são desenvolvidas? & $\mathrm{x}$ & $\mathrm{x}$ \\
\hline \multicolumn{3}{|l|}{ A equipe utiliza o novo Guia Alimentar para a População Brasileira do Ministério da Saúde? } \\
\hline A equipe desenvolve ações com os profissionais do Programa Academia da Saúde? Quais? & $\mathrm{x}$ & \\
\hline $\begin{array}{l}\text { É realizado planejamento conjunto entre a equipe e os profissionais da educação para ações na escola? } \\
\text { Existe documento que comprove? }\end{array}$ & $\mathrm{x}$ & \\
\hline Quais são as estratégias e instrumentos utilizados para monitorar as ações planejadas? & $\mathrm{x}$ & \\
\hline $\begin{array}{l}\text { Qual a principal forma que a equipe utiliza para lidar com as demandas de saúde identificadas entre os } \\
\text { estudantes? }\end{array}$ & $x$ & $\mathrm{x}$ \\
\hline $\begin{array}{l}\text { A equipe utiliza protocolo de acolhimento à demanda espontânea ou linha de cuidado em práticas } \\
\text { integrativas e complementares? }\end{array}$ & $x$ & \\
\hline A equipe identifica famílias elegíveis e encaminha para cadastramento no Programa Bolsa Família? & $\mathrm{x}$ & \\
\hline A equipe registra os dados de acompanhamento dos beneficiários do Programa Bolsa Família? & $\mathrm{x}$ & \\
\hline $\begin{array}{l}\text { A equipe realiza acompanhamento dos casos graves que precisam de atendimento em outros pontos de } \\
\text { atenção? Existe documento que comprove? }\end{array}$ & $x$ & $x$ \\
\hline A equipe realiza avaliação de satisfação do usuário? & $\mathrm{x}$ & $\mathrm{x}$ \\
\hline A equipe dispõe de canais de comunicação para usuários? Existe documento que comprove? Quais? & $\mathrm{x}$ & $\mathrm{x}$ \\
\hline A equipe discute internamente e com a gestão as demandas e reclamações dos usuários? & $x$ & $x$ \\
\hline
\end{tabular}

ACS: agentes comunitários de saúde; HAS: hipertensão arterial sistêmica; PMAQ-AB: Programa Nacional de Melhoria do Acesso e da Qualidade da Atenção Básica; UBS: unidades básicas de saúde.

Fonte: elaboração própria. 


\section{Resultados}

O estudo incluiu 38.865 equipes que participaram do 3o ciclo do PMAQ-AB. Foram analisados os resultados de 35.350 equipes de saúde da família que relataram ter realizado alguma atividade de coordenação do cuidado. Observa-se, pela Tabela 1, que há diferença significativa $(\mathrm{p}<0,001)$, teste qui-quadrado, entre os níveis de coordenação para os instrumentos (PMAQ-AB, Atlas e Observatório), sendo que o maior percentual de equipes encontra-se entre o nível alto e médio de coordenação nos três instrumentos analisados, PMAQ-AB (56,07\% e 38,35\%), Atlas (52,63\% e 40,66\%) e o Observatório (44,82\% e 43,98\%) (Tabela 1).

$\mathrm{Na}$ comparação dos indicadores entre a coordenação do cuidado, houve diferença significativa (teste de Kruskal-Wallis: $\mathrm{p}<0,001)$ entre as tipologias, ou seja, pelo menos um instrumento difere dos demais PMAQ-AB (média: 0,742), Atlas (média: 0,731), Observatório (média: 0,700). O teste de Nemenyi permitiu verificar que todas as tipologias diferem entre si, isto é, a categoria PMAQ difere do Observatório $(\mathrm{p}<0,001)$ e Atlas $(\mathrm{p}<0,001)$, assim como há distinção entre Observatório e Atlas $(\mathrm{p}<0,001)$.

Ao detalhar as tipologias por estrato, por meio do teste qui-quadrado, observou-se que houve associação significativa $(\mathrm{p}<0,001)$ entre os estratos e os níveis de coordenação (Tabela 2). Na análise para o país, observa-se que, na tipologia do PMAQ- $\mathrm{AB}$, todos os estratos apresentaram os maiores percentuais das equipes entre o nível alto e médio de coordenação. Pela Tabela 2, considerando o Atlas, na média coordenação, o estrato 1 apresentou maior percentual (43,81\%). No Observatório, predominou o alto nível, com maior destaque para o $6(51,94 \%)$. Nos estratos 1 e 2 , o maior percentual de equipes estava com médio nível (49,08\% e 46,59\%) e, no nível baixo, o estrato 2 apresentou o maior percentual (13\%).

Na comparação entre os indicadores de coordenação do cuidado por estratos, o teste de KruskalWallis mostrou que pelo menos um estrato difere dos demais $(\mathrm{p}<0,001)$, entre os instrumentos utilizados (Tabela 3).

Ao utilizar o teste de comparações múltiplas de Nemenyi, nota-se que o estrato 6 se distingue de todos os outros ( $\mathrm{p}<0,001)$; o estrato 1 difere de todos $(\mathrm{p}<0,001)$, exceto do estrato $2(\mathrm{p}>0,05)$, padrão este que se comporta de forma semelhante para as três tipologias (PMAQ-AB, Observatório e Atlas). Pode-se afirmar que, na tipologia PMAQ-AB, o estrato 6 possui $62,6 \%$ das equipes com alto grau de coordenação, enquanto os estratos 1 e 2 possuem $52,6 \%$ e 52,4\%, respectivamente (Tabela 4).

\section{Tabela 1}

Níveis de coordenação do cuidado no Brasil. Programa Nacional de Melhoria do Acesso e da Qualidade da Atenção Básica (PMAQ-AB), Brasil, 2018.

\begin{tabular}{lccc}
\hline Dados/Níveis de coordenação & $\mathbf{n}$ & $\%$ & Valor de $\mathbf{p}$ * \\
\hline PMAQ-AB & & & \\
$\quad$ Coordenação baixa & 2.084 & 5,58 & $<0,001$ \\
$\quad$ Coordenação média & 14.325 & 38,35 & \\
Coordenação alta & 20.941 & 56,07 & $<0,001$ \\
Atlas & & & \\
Coordenação baixa & 2.506 & 6,71 & \\
Coordenação média & 15.187 & 40,66 & $<0,001$ \\
Coordenação alta & 19.657 & 52,63 & \\
Observatório & & & \\
Coordenação baixa & 4.185 & 11,20 & \\
Coordenação média & 16.425 & 43,98 & \\
Coordenação alta & 16.740 & 44,82 &
\end{tabular}

Fonte: elaboração própria.

* Teste qui-quadrado. 
Tabela 2

Distribuição de equipes e níveis de coordenação do cuidado, por estratos. Programa Nacional de Melhoria do Acesso e da Qualidade da Atenção Básica (PMAQ-AB), Brasil, 2018.

\begin{tabular}{|c|c|c|c|c|c|c|c|}
\hline \multirow[t]{3}{*}{ Dados/Estratos } & \multicolumn{6}{|c|}{ Nível de coordenação } & \multirow[t]{3}{*}{ Valor de $p$ * } \\
\hline & \multicolumn{2}{|c|}{ Baixo } & \multicolumn{2}{|c|}{ Médio } & \multicolumn{2}{|c|}{ Alto } & \\
\hline & $\mathbf{n}$ & $\%$ & $\mathbf{n}$ & $\%$ & $\mathbf{n}$ & $\%$ & \\
\hline \multicolumn{8}{|l|}{ PMAQ-AB } \\
\hline 1 & 241 & 6,11 & 1.630 & 41,30 & 2.076 & 52,60 & $<0,001$ \\
\hline 2 & 345 & 6,74 & 2.086 & 40,78 & 2.684 & 52,47 & \\
\hline 3 & 293 & 4,91 & 2.290 & 38,36 & 3.386 & 56,73 & \\
\hline 4 & 363 & 5,38 & 2.526 & 37,42 & 3.862 & 57,21 & \\
\hline 5 & 279 & 4,23 & 2.536 & 38,46 & 3.779 & 57,31 & \\
\hline 6 & 353 & 4,60 & 2.534 & 33,04 & 4.783 & 62,36 & \\
\hline \multicolumn{8}{|l|}{ Atlas } \\
\hline 1 & 276 & 6,99 & 1.729 & 43,81 & 1.942 & 49,20 & $<0,001$ \\
\hline 2 & 425 & 8,31 & 2.214 & 43,28 & 2.476 & 48,41 & \\
\hline 3 & 361 & 6,05 & 2.463 & 41,26 & 3.145 & 52,69 & \\
\hline 4 & 444 & 6,58 & 2.654 & 39,31 & 3.653 & 54,11 & \\
\hline 5 & 340 & 5,16 & 2.723 & 41,30 & 3.531 & 53,55 & \\
\hline 6 & 409 & 5,33 & 2.700 & 35,20 & 4.561 & 59,47 & \\
\hline \multicolumn{8}{|l|}{ Observatório } \\
\hline 1 & 459 & 11,63 & 1.937 & 49,08 & 1.551 & 39,30 & $<0,001$ \\
\hline 2 & 665 & 13,00 & 2.383 & 46,59 & 2.067 & 40,41 & \\
\hline 3 & 633 & 10,60 & 2.649 & 44,38 & 2.687 & 45,02 & \\
\hline 4 & 711 & 10,53 & 2.918 & 43,22 & 3.122 & 46,25 & \\
\hline 5 & 592 & 8,98 & 2.935 & 44,51 & 3.067 & 46,51 & \\
\hline 6 & 752 & 9,80 & 2.934 & 38,25 & 3.984 & 51,94 & \\
\hline
\end{tabular}

Fonte: elaboração própria.

* Teste qui-quadrado.

\section{Discussão}

O estudo verificou os níveis de coordenação do cuidado na APS no Brasil, a partir dos dados gerados no 3o ciclo do PMAQ-AB, considerando as principais referências internacionais para medição e avaliação do atributo. Foi possível verificar que a maioria das equipes situa-se entre alto e médio nível de coordenação do cuidado, em todos os cenários avaliados e em todas as metodologias utilizadas. Tais achados configuram uma nova realidade para a coordenação do cuidado na APS, ao se considerarem estudos desenvolvidos a partir de dados do primeiro e segundo ciclos do PMAQ-AB. Os achados até então mostravam uma realidade menos favorável, com maior percentual de equipes em baixo nível de coordenação 22,29. Lima et al. 25, ao compararem os resultados do primeiro e segundo ciclos do PMAQ-AB, verificaram que a coordenação do cuidado foi o atributo pior avaliado, devido à dificuldade de acesso a consultas, baixa integração entre os serviços e falhas no compartilhamento de registros.

Estudos recentes que avaliaram os atributos da APS utilizando o PCATool (na versão profissionais) identificaram bons resultados para a coordenação do cuidado em Goiás 19 e Presidente Prudente (São Paulo) 18. Para o Rio de Janeiro, também foi indicada boa avaliação, no entanto problemas foram relatados no processo de transferência de informação entre especialistas e a APS 17. Rolim et al. 30 encontraram um alto escore em Fortaleza (Ceará), considerando a existência de sistemas de informação; já quanto à integração dos cuidados, escore mais baixo foi identificado. Em uma região administrativa do Distrito Federal, a dimensão integração dos cuidados foi bem avaliada, sobretudo quanto ao conhecimento da rede de atenção, marcação de consultas com especialistas, no encaminhamento 
Tabela 3

Comparações dos indicadores de coordenação do cuidado por estrato. Programa Nacional de Melhoria do Acesso e da Qualidade da Atenção Básica (PMAQ-AB), Brasil, 2018.

\begin{tabular}{|c|c|c|c|c|c|c|c|}
\hline Índices/Estratos & $\mathbf{n}$ & Média & Erro padrão & $\begin{array}{c}\text { Q1 } \\
\text { (1ㅇ quartil) }\end{array}$ & $\begin{array}{c}\text { Q2 } \\
\text { (mediana) }\end{array}$ & $\begin{array}{c}\text { Q3 } \\
\text { (3o quartil) }\end{array}$ & Valor de $p$ * \\
\hline \multicolumn{8}{|l|}{ Total } \\
\hline 1 & 3.947 & 0,732 & 0,002 & 0,659 & 0,757 & 0,826 & $<0,001$ \\
\hline 2 & 5.115 & 0,729 & 0,002 & 0,657 & 0,757 & 0,826 & \\
\hline 3 & 5.969 & 0,745 & 0,002 & 0,674 & 0,770 & 0,839 & \\
\hline 4 & 6.751 & 0,746 & 0,002 & 0,673 & 0,772 & 0,842 & \\
\hline 5 & 6.594 & 0,748 & 0,002 & 0,679 & 0,772 & 0,836 & \\
\hline 6 & 7.670 & 0,760 & 0,001 & 0,689 & 0,790 & 0,855 & \\
\hline \multicolumn{8}{|l|}{ Atlas } \\
\hline 1 & 3.947 & 0,721 & 0,002 & 0,643 & 0,747 & 0,821 & $<0,001$ \\
\hline 2 & 5.115 & 0,716 & 0,002 & 0,638 & 0,743 & 0,818 & \\
\hline 3 & 5.969 & 0,732 & 0,002 & 0,656 & 0,758 & 0,832 & \\
\hline 4 & 6.751 & 0,736 & 0,002 & 0,658 & 0,761 & 0,837 & \\
\hline 5 & 6.594 & 0,737 & 0,002 & 0,663 & 0,760 & 0,830 & \\
\hline 6 & 7.670 & 0,751 & 0,001 & 0,676 & 0,781 & 0,849 & \\
\hline \multicolumn{8}{|l|}{ Observatório } \\
\hline 1 & 3.947 & 0,684 & 0,002 & 0,592 & 0,708 & 0,800 & $<0,001$ \\
\hline 2 & 5.115 & 0,684 & 0,002 & 0,592 & 0,712 & 0,800 & \\
\hline 3 & 5.969 & 0,703 & 0,002 & 0,612 & 0,729 & 0,817 & \\
\hline 4 & 6.751 & 0,706 & 0,002 & 0,612 & 0,733 & 0,821 & \\
\hline 5 & 6.594 & 0,707 & 0,002 & 0,620 & 0,733 & 0,814 & \\
\hline 6 & 7.670 & 0,721 & 0,002 & 0,634 & 0,754 & 0,833 & \\
\hline
\end{tabular}

Fonte: elaboração própria.

* teste de Kruskal-Wallis.

e nas transferências de responsabilidade. No entanto foram identificados problemas nas trocas de informações e possíveis falhas nos registros 31 .

Vários estudos mensuraram o comportamento da coordenação do cuidado em diversos contextos. Trabalhos utilizando o PCATool apresentaram altos escores quando considerada a opinião de usuários, no Rio Grande do Sul 16 e em Manaus (Amazonas) 32. No Pará, foi encontrado alto escore na coordenação de sistemas de informação e escore baixo na coordenação de integração do cuidado 33 . Silva \& Alves 34 , por outro lado, ao estudarem o grau de implantação dos atributos da APS, verificaram escores distintos entre usuários e profissionais no atendimento às crianças nas áreas rurais e urbanas em Diamantina (Minas Gerais). Tanto profissionais como usuários avaliaram positivamente o subitem sistema de informação, já a integração dos cuidados, apresentou avaliação ruim pelos usuários.

De forma geral, ao longo dos anos, é possível verificar uma evolução da coordenação do cuidado no país, como fruto dos esforços realizados para o fortalecimento da APS: ampliação do acesso por meio da expansão das EqSF, a melhoria na qualidade dos atendimentos, a capacitação dos profissionais para atuação multidisciplinar, o Programa Mais Médicos, alocação de tecnologias para melhorar a resolutividade, entre outros 35,36 . Almeida et al. 20 afirmam que formulações como a Política Nacional de Atenção Básica (PNAB, 2011), Núcleos de Apoio à Saúde da Família (NASF), a construção das Redes de Atenção à Saúde (RAS) e o PMAQ-AB foram importantes na conformação de uma melhor coordenação.

Tais ações e políticas conduziram à incorporação de elementos fundamentais para a realização de atividades de coordenação do cuidado, como a implantação de prontuários eletrônicos, disponibilidade de internet, computadores e instalação de telefones nas unidades de saúde, sistema intermunicipal 
Tabela 4

Comparações múltiplas dos indicadores de coordenação do cuidado, por estratos. Programa Nacional de Melhoria do Acesso e da Qualidade da Atenção Básica (PMAQ-AB), Brasil, 2018.

\begin{tabular}{cccccc}
\hline Dados/Estratos & $\mathbf{1}$ & $\mathbf{2}$ & $\mathbf{3}$ & $\mathbf{4}$ & $\mathbf{5}$ \\
\hline PMAQ-AB & & & & \\
2 & 0,997 & & & \\
3 & 0,000 & 0,000 & & \\
4 & 0,000 & 0,000 & 0,968 & 0,000 \\
5 & 0,000 & 0,000 & 0,995 & 1,000 & \\
6 & 0,000 & 0,000 & 0,000 & 0,000 & \\
Atlas & & & & & \\
2 & 0,959 & & & & \\
3 & 0,001 & 0,000 & 0,000 & \\
4 & 0,000 & 0,000 & 0,639 & & \\
5 & 0,000 & 0,000 & 0,920 & 0,995 & \\
6 & 0,000 & 0,000 & 0,000 & 0,000 & \\
Observatório & & & & & \\
2 & 0,995 & 0,000 & & \\
3 & 0,000 & 0,000 & & 0,000 & \\
4 & 0,000 & 0,000 & 0,850 & & \\
5 & 0,000 & 0,000 & 0,916 & & \\
6 & 0,000 & 0,000 & 0,000 & & \\
\hline
\end{tabular}

Nota: teste de Nemenyi.

Fonte: elaboração própria.

de transporte de pacientes, expansão e implementação das centrais de regulação, incorporação do NASF e incentivo às melhorias nos mecanismos de referência e contrarreferência 20.

É importante salientar que a manutenção destes resultados, como de tantos outros, dentre os quais a redução da mortalidade infantil e a melhoria no acesso a serviços e atendimentos, obtidos por meio das ações de fortalecimento da APS, dependem de políticas e programas de saúde que continuem sendo executados 35 . No entanto a atual crise política e econômica vivenciada pelo país no final da última década vem causando uma progressiva redução nos investimentos em saúde, resultado de medidas de austeridade fiscal que, a curto e longo prazo, comprometerão os avanços conquistados pelo Sistema Único de Saúde 37.

No que se refere à análise da coordenação nos estratos, os níveis mais altos ocorreram em todos os estratos. Foi observado que o estrato 6 possuía os níveis mais altos, diferindo dos demais. E os estratos 1 e 2 apresentaram níveis mais baixos, com padrões similares. Tais achados foram observados para todos os instrumentos utilizados, com pouca variação entre eles. Os resultados chamam atenção pelo fato dos estratos serem diretamente proporcionais à densidade populacional e às condições socioeconômicas. Melhores resultados são encontrados em municípios maiores, onde existe maior concentração de profissionais de saúde, equipamentos diagnósticos, maior disponibilidade de meios de comunicação e locomoção, chamando atenção para a intensa desigualdade locorregional existente no Brasil.

Segundo dados do Instituto Brasileiro de Geografia e Estatística (IBGE) 38, 68,2\% dos municípios brasileiros têm menos de 20 mil habitantes. Nessas localidades, a coordenação do cuidado se torna um importante mecanismo para a redução das desigualdades e no enfrentamento das iniquidades em saúde ${ }^{39}$. Sendo um dispositivo potente de atuação, pode fazer o diferencial em locais onde o sistema de saúde ainda é precário, sobretudo na oferta de média e alta complexidade, o que conduz à necessidade de encaminhamento aos grandes centros para tratamentos complexos. Medeiros \& Gerhardt 40 confirmam essa mesma realidade no Rio Grande do Sul e verificam deficiência na construção das RAS 
devido à dificuldade de acesso e de organização dos serviços. Turci et al. 41 apontam que a construção da rede regionalizada de saúde é uma estratégia para o enfrentamento das diversidades territoriais do país, e é apontado como um dos recursos mais eficientes para obter melhores resultados para a coordenação do cuidado.

Adicionalmente, este fator é agravado na medida em que a maior parte dos municípios de pequeno porte está localizada nas regiões mais pobres, onde há, ainda, escassez de recursos tecnológicos e de comunicação. Sem incorporação de prontuário eletrônico e tecnologias de informação e comunicação (TIC), que facilitam a relação entre APS e demais níveis da atenção, barreiras são colocadas para o avanço na coordenação do cuidado. A APS como componente estratégico da organização dos serviços de saúde segue, portanto, dependente da articulação dos recursos essenciais para o enfrentamento de problemas de saúde em todos os seus níveis de complexidade 20.

Os resultados deste estudo evidenciaram que os níveis de coordenação do cuidado diferem entre os três instrumentos utilizados, ou seja, o PMAQ-AB, o Atlas e o Observatório. Os achados chamam atenção e evidenciam a necessidade de estudos mais detalhados que permitam identificar quais fatores, de forma específica, promoveram tais diferenças. Contudo é possível concluir que os três instrumentos convergem para um mesmo sentido.

Cabe ainda ressaltar que o presente estudo consistiu em uma tentativa de correlacionar as referências internacionais à realidade brasileira, de forma a balizar o PMAQ-AB como uma ferramenta eficaz na mensuração do atributo. Tentativas semelhantes são relatadas na literatura nos mais variados ambientes de cuidado.

Considerando o Atlas, recente revisão de literatura identificou quatorze estudos publicados entre 2015 e 2020 que o utilizaram como estrutura para o estudo da coordenação do cuidado em distintas realidades 42 . Esta revisão constatou que a coordenação reduz a fragmentação e os custos da assistência e melhora a qualidade da assistência à saúde. Os programas de cuidados transitórios são um componente importante da coordenação do cuidado eficaz e têm demonstrado reduzir eventos adversos e prevenir reinternações hospitalares.

No cenário da APS foi utilizado para a criação de um instrumento de pesquisa sobre coordenação do cuidado aplicado a profissionais e usuários em Connecticut (Estados Unidos) ${ }^{43}$. A primeira versão do Atlas, publicada em 2010, foi utilizada na Carolina do Norte (Estados Unidos) em pesquisa com profissionais da APS e em hospitais para entender problemas e identificar soluções para a coordenação do cuidado 44 .

O Atlas também foi referência na criação de um instrumento para avaliar, na perspectiva dos usuários, a coordenação do cuidado no tratamento de câncer nos Estados Unidos. O instrumento foi bem avaliado, sendo considerado uma alternativa para balizar estratégias de coordenação do cuidado 45 .

Contudo, Bynum \& Ross 11 afirmam que o Atlas apresenta algumas limitações ligadas à dificuldade de utilização em larga escala, por envolver pesquisas demoradas e que demandam uma extensa busca e manuseio de documentos. Mesmo com estas características, o Atlas é uma referência, seja por disponibilizar instrumentos como por oferecer um arcabouço teórico que subsidia a criação de dispositivos específicos de acordo com a necessidade dos serviços.

O Observatório, de forma diferente do Atlas não dispõe de instrumentos prontos que possam ser utilizados ou adaptados, mas é uma importante referência, por também oferecer aporte teórico e nortear os estudos sobre coordenação. Os resultados do Observatório foram considerados em uma analise sobre a realidade da saúde espanhola e orientou na identificação de questões a serem trabalhadas como fortalecimento da força trabalho, adequado financiamento da APS e necessidade de estudos para medir o real impacto da APS no país ${ }^{14}$. Na Grécia, as dimensões definidas para avaliar a integração assistencial foram baseadas nos indicadores do Observatório ${ }^{46}$. O trabalho também foi adotado como estrutura de pesquisa em revisão de literatura que avaliou a evolução da APS no Japão 47.

No Reino Unido, revisão sistemática sobre coordenação de cuidado, utilizando os estudos do Observatório, afirma que os cuidados integrados e a experiência do paciente são essenciais para a coordenação e prestação de cuidados sociais e de saúde, mas sua aplicação conjunta é mal compreendida. Conclui que pessoas com necessidades complexas experimentam falta de coordenação entre as equipes e recursos da comunidade 48 . 
Diante das evidencias científicas aqui apresentadas para a coordenação do cuidado no Brasil, é importante observar que cabem questionamentos na comparação entre os resultados do PMAQ-AB e do PCAtool, devido a diferenças conceituais entre os instrumentos. Destaca-se que, ao analisar os resultados de pesquisas desenvolvidas a partir do primeiro e segundo ciclo do PMAQ-AB, observou-se uma evolução significativa do atributo em um curto espaço de tempo. Assim, é necessária a realização de pesquisas mais detalhadas que permitam identificar quais foram os reais fatores que influenciaram ou determinaram essa mudança.

Este estudo apresentou algumas limitações: as tipologias foram construídas a partir de um instrumento já em processo de utilização, não permitindo que todo o potencial das concepções de coordenação avaliadas fosse explorado em toda sua plenitude. O 3o ciclo do PMAQ-AB contou com poucas questões nas quais se exigia uma comprovação das afirmações realizadas, podendo, portanto, permitir uma superestimação do que efetivamente tem ocorrido na APS no Brasil. As respostas foram fornecidas pelos profissionais de saúde, e não a partir de instrumentos precisos de aferição.

\section{Conclusão}

Sendo a coordenação do cuidado um dos alicerces para APS forte e eficaz, conhecer o perfil de funcionamento nos serviços de saúde norteia o aprimoramento e a criação de políticas e ações.

No presente estudo, a coordenação do cuidado na APS no Brasil apresentou um predomínio de equipes com altos e médios níveis em todos os cenários, considerando os três instrumentos utilizados. Os achados configuram uma evolução do atributo, que pode ser reconhecido como resultado do desenvolvimento da APS nos últimos anos. Também foram constatadas diferenças significativas dos níveis de coordenação entre os estratos em todas as tipologias, com os menores municípios apresentando níveis mais baixos de coordenação.

O bom desempenho do atributo de coordenação reconhecido neste estudo soma-se a outros trabalhos que demonstram apreensão diante do cenário de incertezas e ameaças ao futuro da APS no país. Às mudanças na Política Nacional da Atenção Básica em 2017, que já direcionaram para a abolição da prioridade para a EqSF, acrescenta-se, em 2019, a Portaria no 2.979/2019 49, que altera radicalmente a bases de financiamento, potencializando a desfiguração da APS, que passa a ter um perfil voltado para a oferta de serviços básicos de saúde seletivos, organizados sob a lógica de atendimento "queixa-conduta".

No contexto de avaliação da APS e de forma específica do atributo coordenação do cuidado, o PMAQ-AB se mostrou uma boa alternativa quando comparado ao Atlas de Medidas de Coordenação do Cuidado e ao Observatório Europeu de Políticas e Sistemas de Saúde. No entanto foi constatado que os níveis de coordenação do cuidado obtidos diferem entre os três instrumentos, o que sinaliza para a importância de novos estudos que busquem identificar os fatores que conduziram a tais achados. 


\section{Colaboradores}

M. J. B. Cruz, A. F. Santos, C. Macieira, D. M. X. Abreu, A. T. G. M. Machado e E. I. G. Andrade contribuíram na concepção e projeto de estudo, na análise e interpretação dos dados, na redação do artigo e revisão crítica relevante do conteúdo intelectual, na aprovação final da versão a ser publicada e são responsáveis por todos os aspectos do trabalho na garantia da exatidão e integridade de qualquer parte da obra.

\section{Informações adicionais}

ORCID: Maria Jesus Barreto Cruz (0000-00032735-3909); Alaneir de Fátima dos Santos (00000002-7674-0449); César Macieira (0000-00023238-4489); Daisy Maria Xavier de Abreu (00000002-6855-8612); Antônio Thomaz Gonzaga da Matta Machado (0000-0002-0516-8529); Eli Iola Gurgel Andrade (0000-0002-0206-2462).

\section{Agradecimentos}

À Coordenação de Aperfeiçoamento de Pessoal de Nível Superior (CAPES) pelo financiamento da bolsa de doutorado de M. J. B. Cruz.

\section{Referências}

1. Schang L, Waibel S, Thomson S. Measuring care coordination: health system and patient perspectives. London: LSE Health; 2013.

2. Kumpunen S, Edwards N, Georghiou T, Hughes G. Why do evaluations of integrated care not produce the results we expect? Int J Care Coord 2020; 23:9-13.

3. Starfield B. Atenção primária: equilibrio entre necessidade de saúde, serviços e tecnologia. Brasília: Organização das Nações Unidas para a Educação, a Ciência e a Cultura/Ministério da Saúde; 2004.

4. McDonald KM, Schultz E, Albin L, Pineda N, Lonhart J, Sundaram V, et al. Care Coordination Measures Atlas update. https://www. ahrq.gov/ncepcr/care/coordination/atlas.html (acessado em 26/Jan/2018).

5. Dessers E, Mohr BJ. Uma perspectiva do ecossistema na coordenação do cuidado: lições de campo. Int J Care Coord 2020; 23:5-8.

6. Bodenheimer T, Chen E, Bennett HD. Confronting the growing burden of chronic disease: can the U.S. health care workforce do the job? Health Aff (Millwood) 2009; 28:64-74.

7. Hays RD, Martino S, Brown JA, Cui M, Cleary P, Gaillot S, et al. Evaluation of a Care Coordination Measure for the Consumer Assessment of Healthcare Providers and Systems (CAHPS) Medicare survey. Med Care Res Rev 2014; 71:192-202.

8. Kringos DS, Boerma WGW, Hutchinson A, Saltman RB. Building primary care in a changing Europe. Copenhagen: European Observatory on Health Systems and Policies/World Health Organization; 2015.

9. Berkowitz SA, Parashuram S, Rowan K, Andon L, Bass EB, Bellantoni M, et al. Association of a Care Coordination Model with health care costs and utilization: the Johns Hopkins Community Health Partnership (J-CHiP). JAMA Netw Open 2; 1:e184273.

10. Weeks DL, Polello JM, Hansen DT, Keeney BJ, Conrad DA. Measuring primary care organizational capacity for diabetes care coordination: the diabetes care coordination readiness assessment. J Gen Intern Med 2014; 29:98103.

11. Bynum JPW, Ross JS. A measure of care coordination? J Gen Intern Med 2013; 28:336-8.

12. World Health Organization. Continuity and coordination of care: a practice brief to support implementation of the WHO Framework on integrated people-centred health services. Geneva: World Health Organization; 2018.

13. Agency for Healthcare Research and Quality. Care Coordination Quality Measure for Primary Care (CCQM-PC). https://www.ahrq. gov/ncepcr/care/coordination/quality/index. html (acessado em 19/Jul/2021).

14. Vicente VC. Construyendo la atención primaria española en una Europa cambiante. Aten Primaria 2016; 48:71-2. 
15. Seys D, Panella M, VanZelm R, Sermeus W, Aeyels D, Bruyneel L, et al. Care pathways are complex interventions in complex systems: New European Pathway Association framework. Int J Care Coord 2019; 22:5-9.

16. Bandeira D, Damaceno AN, Weiller TH, Lopes LFD. Evaluation of the coordination of care by users of primary health care services. REME Rev Min Enferm 2020; 24:e-1278.

17. Paixão TM, Sousa AI, Souza MHDN, Farias SNP. Coordenação da atenção primária: limites e possibilidades para a integração do cuidado. Rev Enferm UERJ 2019; 27:e42655.

18. Gomes, MFP, Fracolli LA. Evaluation of the Family Health Strategy in the region of Presidente Prudente-SP, Brazil. Mundo Saúde 2019; 43:306-25.

19. Maia LG, Silva LA, Guimarães RA, Pelazza BB, Leite GR, Barbosa MA, et al. A qualidade de serviços de atenção primária, a formação profissional e o Programa Mais Médicos em uma região de saúde do sudoeste goiano. Rev Bras Epidemiol 2020; 23:e200014.

20. Almeida PF, Medina MG, Fausto MCR, Giovanella L, Bousquat A, Mendonça MHM. Coordenação do cuidado e atenção primária à saúde no Sistema Único de Saúde. Saúde Debate 2018; 42(N spe 1):244-60.

21. Ministério da Saúde. Saúde mais perto de você - acesso e qualidade programa nacional de melhoria do acesso e da qualidade da atenção básica (PMAQ). Manual Instrutivo. http:// multiresidencia.com.br/bibliotecas/cadernosde-atencao-basica/abcad35.pdf (acessado em 18/Jun/2020).

22. Souza MF, Santos ADF, Reis IA, Santos MAC, Jorge ADO, Machado ATGM, et al. Coordenação do cuidado no PMAQ-AB: uma análise baseada na Teoria de Resposta ao Item. Rev Saúde Pública 2017; 51:87.

23. Fausto MCR, Giovanella L, Mendonça MHM, Fonseca HMS, Lima JG. A posição da Estratégia Saúde da Família na rede de atenção à saúde na perspectiva das equipes e usuários participantes do PMAQ-AB 2012. Saúde Debate 2014; 38(N spec):13-33.

24. Chang L, Wanner KJ, Kovalsky D, Smith KL, Rhodes KV. "It's really overwhelming": patient perspectives on care coordination. J Am Board Fam Med 2018; 31:682-90.

25. Lima JG, Giovanella L, Fausto MCR, Bousquat A, Silva EV, Lima JG, et al. Atributos essenciais da Atenção Primária à Saúde: resultados nacionais do PMAQ-AB. Saúde Debate 2018; 42(N spec 1):52-66.

26. Agresti A, Kateri M. Categorical data analysis. Gainesville: John Wiley; 2011.

27. Anderson TW, Darling DA. Tests of goodnessof-fit. In: Lovric M, editor. International encyclopedia of statistical science. Berlin: SpringerVerlag; 2011. p. 52-4.

28. Hollander M, Wolfe DA. Nonparametric statistical methods. 2nd Ed. New York: John Wiley \& Sons; 1999.
29. Cruz MJB, Santos AF, Araújo LHL, Andrade EIG. A coordenação do cuidado na qualidade da assistência à saúde da mulher e da criança no PMAQ. Cad Saúde Pública 2019; 35:e00004019.

30. Rolim LB, Monteiro JG, Meyer APGFV, Nuto SAS, Araújo MFM, Freitas RWJF, et al. Avaliação dos atributos da Atenção Primária à Saúde de Fortaleza, Ceará, Brasil. Rev Bras Enferm 2019; 72:19-26.

31. Leandro SS, Monteiro PS. Avaliação da coordenação da atenção na perspectiva dos profissionais de saúde. Enferm Foco (Brasília) 2017; 8:65-9.

32. De Araújo Vieira Sá LYB. A avaliação da atenção primária: um olhar preliminar através do PCATool em Manaus, Amazonas. APS em Revista 2019; 1:98-111.

33. De Souza BR, Tavares JB, Pinto Girard CC, Ferreira IP. Avaliação da Atenção Primária à Saúde em uma estratégia saúde da família no interior do Pará: utilização do PCATool-versão Brasil. APS em Revista 2019; 1:112-20.

34. Silva GS, Alves CRL. Avaliação do grau de implantação dos atributos da atenção primária à saúde como indicador da qualidade da assistência prestada às crianças. Cad Saúde Pública 2019; 35:e00095418.

35. Tasca R, Massuda A, Carvalho WM, Buchweitz C, Harzheim E. Recomendações para o fortalecimento da atenção primária à saúde no Brasil. Rev Panam Salud Pública 2020; 44:e4.

36. Macinko J, Mendonça CS. Estratégia Saúde da Família, um forte modelo de Atenção Primária à Saúde que traz resultados. Saúde Debate 2018; 42(N spe 1):18-37.

37. Massuda A, Hone T, Leles FAG, de Castro MC, Atun R. The Brazilian health system at crossroads: progress, crisis and resilience. BMJ Glob Health 2018; 3:e000829.

38. Instituto Brasileiro de Geografia e Estatística. IBGE divulga as estimativas da população dos municípios para 2019. Agência IBGE Notícias 2019; 28 ago. https://agenciadenoticias.ibge. gov.br/agencia-sala-de-imprensa/2013-agen cia-de-noticias/releases/25278-ibge-divulga -as-estimativas-da-populacao-dos-municipios -para-2019.

39. Ribeiro SP, Cavalcanti MLT. Atenção primária e Coordenação do Cuidado: dispositivo para ampliação do acesso e a melhoria da qualidade. Ciênc Saúde Colet 2020; 25:1799-808.

40. Medeiros CRG, Gerhardt TE. Avaliação da Rede de Atenção à Saúde de pequenos municípios na ótica das equipes gestoras. Saúde Debate 2015; 39(N spec):160-70.

41. Turci MA, Lima-Costa MF, Macinko J. Influência de fatores estruturais e organizacionais no desempenho da atenção primária à saúde em Belo Horizonte, Minas Gerais, Brasil, na avaliação de gestores e enfermeiros. Cad Saúde Pública 2015; 31:1941-52. 
42. Chakurian D, Popejoy L. Utilizing the care coordination Atlas as a framework: an integrative review of transitional care models. Int J Care Coord 2021; 24:57-71.

43. Zlateva I, Anderson D, Coman E, Khatri K, Tian T, Fifield J. Development and validation of the Medical Home Care Coordination Survey for assessing care coordination in the primary care setting from the patient and provider perspectives. BMC Health Serv Res 2015; 15:226.

44. Jones CD, Vu MB, O’Donnell CM, Anderson ME, Patel S, Wald HL, et al. A failure to communicate: a qualitative exploration of care coordination between hospitalists and primary care providers around patient hospitalizations. J Gen Intern Med 2015; 30:417-24.

45. Okado I, Cassel K, Pagano I, Holcombe RF. Development and psychometric evaluation of a questionnaire to measure cancer patients' perception of care coordination. BMC Health Serv Res 2020; 20:52.
46. Sifaki-Pistolla D, Chatzea VE, Markaki A, Kritikos K, Petelos E, Lionis C. Operational integration in primary health care: patient encounters and workflows. BMC Health Serv Res 2017; 17:788.

47. Kato D, Ryu H, Matsumoto T, Abe K, Kaneko $\mathrm{M}$, Ko M, et al. Building primary care in Japan: literature review. J Gen Fam Med 2019; 20:170-9.

48. Davidson L, Scott J, Forster N. Patient experiences of integrated care within the United Kingdom: a systematic review. Int J Care Coord 2021; 24:39-56.

49. Ministério da Saúde. Portaria no 2.979, de 12 de novembro de 2019. Institui o Programa Previne Brasil, que estabelece novo modelo de financiamento de custeio da Atenção Primária à Saúde no âmbito do Sistema Único de Saúde, por meio da alteração da Portaria de Consolidação no 6/GM/MS, de 28 de setembro de 2017. Diário Oficial da União 2019; 13 nov. 
Abstract

This study aimed to compare the results obtained with the coordination of care through the Brazilian National Program for Improvement of Access and Quality of Basic Care (PMAQ-AB), with the parameters adopted by the Care Coordination Measures Atlas and the European Observatory on Health Systems and Policies. A cross-sectional study was performed using the dataset from the third cycle of the PMAQ-AB. Three typologies of coordination of care were created: $P M A Q-A B$, Atlas, and Observatory. Chi-square test was applied to compare proportions and Kruskal-Wallis and Nemenyi tests to verify and identify potential differences between the typologies. Significance was set at 5\%. In all, 35,350 teams were assessed that performed some activity in care coordination. A significant difference was observed $(p<0.001)$ between levels of coordination, with a higher percentage between the high and medium levels in the three instruments, PMAQ-AB $(56.07 \%$ and 38.35\%), Atlas (52.63\% and 40.66\%), and Observatory (44.82\% and $43.98 \%$ ). In the comparison of the indicators, there was a significant difference $(p<0.001)$ between the typologies. For Brazil, in the PMAQ-AB typology, all the strata displayed a higher percentage between the high and medium levels; in the Atlas, stratum 1 stood out in the medium level (43.81\%); the high level predominated in the Observatory. In the comparison of the indicators by strata, at least one stratum differed from the others $(p<0.001)$. Number 6 differed from the others $(p<0.001)$, and number 1 differed from all of them $(p<0.001)$ except number $2(p$ $>0.05$ ). The levels of coordination of care differed according to the instruments used. High and medium levels were identified, showing the need for additional studies.

Health Care Quality, Access, and Evaluation; Primary Health Care; Comparative Study; Health Care Quality Assurance; Health Services Research

\section{Resumen}

El objetivo de este estudio fue comparar los resultados obtenidos para la coordinación del cuidado, a partir del Programa Nacional de Mejoría de Acceso y Calidad de la Atención Básica (PMAQ-AB), con los parámetros adoptados por el Atlas de Medidas de Coordinación del Cuidado y por el Observatorio Europeo de Políticas y Sistemas de Salud. Se realizó un estudio transversal, basado en el banco de datos del 3er ciclo del PMAQ-AB. Se crearon tres tipologías de coordinación del cuidado: PMAQ-AB, Atlas y Observatorio. El test de chi-cuadrado se aplicó para comparar las proporciones, el test de Kruskal-Wallis y el de Nemenyi para verificar e identificar eventuales diferencias entre las tipologías. El nivel de significancia fue de 5\%. Se evaluaron a 35.350 equipos que realizaron alguna actividad de coordinación del cuidado. Se observó una diferencia significativa $(p<0,001)$, entre los niveles de coordinación, con mayor porcentaje entre el nivel alto y medio en los tres instrumentos, PMAQ-AB (56,07\% y 38,35\%), Atlas $(52,63 \%$ y 40,66\%) y el Observatorio $(44,82 \%$ y 43,98\%). En la comparación de los indicadores, hubo una diferencia significativa $(p<0,001)$ entre las tipologías. Para Brasil, en la tipología PMAQ-AB todos los estratos expusieron un mayor porcentaje entre el nivel alto y medio; en el Atlas, el estrato 1 se destacó en el nivel medio $(43,81 \%)$ y, en el $\mathrm{Ob}$ servatorio, predominó el nivel alto. En la comparación de los indicadores por estratos, por lo menos un estrato difirió de los demás $(p<0,001)$. El 6 se distinguió de los demás $(p<0,001)$, y el 1 difirió de todos $(p<0,001)$, excepto del $2(p>0,05)$. Los niveles de coordinación del cuidado se diferenciaron entre los instrumentos utilizados. Se identificaron niveles altos y medios, demostrando la necesidad de estudios adicionales.

Calidad, Acceso y Evaluación de la Atención de Salud; Atención Primaria de Salud; Estudio Comparativo; Garantía de la Calidad de Atención de Salud; Investigación sobre Servicios de Salud

Recebido em 03/Abr/2021

Versão final reapresentada em 05/Ago/2021

Aprovado em 09/Ago/2021 